\title{
Bat algorithm and k-means techniques for classification performance improvement
}

\author{
Rozlini Mohamed, Munirah Mohd Yusof, Noorhaniza Wahid, Norhanifah Murli, Muhaini Othman \\ Faculty of Computer Science \& Information Technology, Universiti Tun Hussein Onn Malaysia, Malaysia
}

\begin{tabular}{l}
\hline \hline Article Info \\
\hline Article history: \\
Received Jan 9, 2019 \\
Revised Mar 2, 2019 \\
Accepted Mar 18, 2019 \\
\hline
\end{tabular}

\section{Keywords:}

Bat algorithm

Classification

Discretization

Feature selection

K-Means

\begin{abstract}
This paper presents Bat Algorithm and K-Means techniques for classification performance improvement. The objective of this study is to investigate efficiency of Bat Algorithm in discrete dataset and to find the optimum feature in discrete dataset. In this study, one technique that comprise the discretization technique and feature selection technique have been proposed. Our contribution is in two process of classification: pre-processing and feature selection process. First, to proposed discretization techniques called as BkMD, where we hybrid Bat Algorithm technique and K-Means classifier. Second, to proposed BkMDFS as feature selection technique where Bat Algorithm is embed into BkMD. In order to evaluate our proposed techniques, 14 continuous dataset from various applications are used in experiment. From the experiment, results show that BkMDFS outperforms in most performance measures. Hence it shows that, Bat Algorithm have potential to be one of the discretization technique and feature selection technique.
\end{abstract}

Copyright $\odot 2019$ Institute of Advanced Engineering and Science. All rights reserved.

\section{Corresponding Author:}

Rozlini Binti Mohamed

Faculty of Computer Science \& Information Technology,

Universiti Tun Hussein Onn Malaysia,

86400 Parit Raja, Batu Pahat, Johor, Malaysia.

Email: rozlini@uthm.edu.my

\section{INTRODUCTION}

Classification is one of the important area in data mining. The main process in classification are preprocessing and feature selection. These process may influence the classification performance. There are many ways to perform pre-processing and feature selection including discretization during pre-processing step. Discretization is the process to transform the continuous value into integer value or known as discrete [1]. The techniques for discretization can be categorized in two perspectives, supervised and unsupervised [2]. The goal of data discretization is to determine the best set of break point to group the data. Break point is a limit of an interval of integer values. In classification, the goal of discretization is to make a better classification by yielding higher accuracy [3]. Discretization can also be useful for data cleansing tasks including missing value imputation and corrupt data detection (CDD). From García-Gil in 2018 [4], have propose a new ensemble method based on PCA for the dimensionality reduction step and Random Discretization. Discretization also used in missing value imputation techniques such as FIMUS [5] and CDD techniques such as CAIRAD [6] rely on discretization algorithms. Some data mining techniques require discrete data rather than continuous data and it is very important to have algorithms for discretization of continuous data [7].

Discretization algorithms have been intensively researched and have been developed embedded with various techniques. The use of discretization algorithm has been in different purpose and application. There are many algorithm are used to perform discretization such as statistical technique based on Chi-square 
statistic [8], heuristic algorithm [9, 10] and K-Means classifier [11]. K-Means classifier with Rough set was used by Xing et al. in [12] to transform continuous data into continuous data. Tahir et al. in [13] using KMeans in discretization technique to proposed method that able to generate better detection rate and accuracy.

Feature selection can lead to getting good classification accuracy. Feature selection is a process to identify the relevant feature in dataset. After the process, selected features might be possibility half of the size of the original feature dataset. Bat Algorithm (BA) is used to find the optimal features in [14, 15]. Since, BA is a powerful technique to optimize the number of features, this algorithm is combined with another technique such as rough set $[16,17]$. BA, is one of the Swarm Intelligent algorithm initiated by Yang that more powerful compare to the other evolutionary algorithm [18].

Nowadays, many researchers have extended the original BA to solve discrete problem such as [19] in fault system diagnosis [20], Traveling Salesman Problem (TSP) [21]. Krause et. al. [22], also applied bat to handle discrete problems. In 2014, Nakamura et al. [23], introduced binary bat algorithm to solve feature selection problems. The main idea is to associate each bat a set of binary coordinates that denote whether a feature will belong to the final set of features or not. Since the problem is to select or not a given feature, the bat's position is then represented by binary vectors. Enache and Sgarciu in [24] proposed a feature selection technique in transforming the continuous data into discrete data for instruction detection application.

The objective of this study is to investigate efficiency of BA in discrete dataset and to find the optimum feature in discrete dataset. In order to prove the efficiency of BA, this study proposed one technique that comprise the discretization technique and feature selection technique have been proposed. Our contribution in two process of classification. First, we contribute in pre-processing process by look the benefit of K-Means and BA technique. BA is an optimization technique which is used to select the optimal feature from a dataset. But, the drawback of BA is not capable to convert data into discrete. Since, K-Means classifier has the ability to discretize the data, BA and K-Means are combined as a discretization technique, known as BkMD. Second, we apply BA as feature selection technique called as BkMDFS. BkMDFS is one technique that comprises discretization technique (BkMD) and feature selection technique (BA).

The proposed techniques, BkMD BkMDFS are evaluated and compared with the existing techniques by applying them on simulated in various application datasets. The result show that the proposed techniques leads to considerably better accuracy results. The merit of this study is heuristic and random search that based on an objective criterion to find the optimize feature in dataset.

Literature review that has been done author used in the chapter "Introduction" to explain the difference of the manuscript with other papers, that it is innovative, it are used in the chapter "Research Technique" to describe the step of research and used in the chapter "Results and Discussion" to support the analysis of the results. The conclusion will be discussed in the chapter "Conclusion".

\section{RESEARCH TECHNIQUE}

\subsection{Dataset and Experimental Settings}

There are 14 datasets were used to validate study. Features in these datasets are of numerical only which are each dataset must have more than half continuous features. The experiments were conducted using 14 datasets from different applications; Credit Approval (Ds1), Ecoli (Ds2), Hill Valley (Ds3), Image Segmentation (Ds4), Libras Movement (Ds5), Plant Species (Ds6), Steel Plates Faults (Ds7), Urband Land (Ds8), Automobile (Ds9), Abalone (Ds10), Yeast (Ds11), Waveform (Ds12), Ionosphere (Ds13) and Water Treatment (Ds14). These datasets called as original datasets (ODS) that obtained from UCI Machine Learning Repository [UCI] that public available for research. Number of instances in dataset ranges from 159 to 5000. Dataset vary from 8 to 100 features. All information about the datasets used in this study are shown in Table 1. Meanwhile, Figure 1 illustrates framework BkMDFS to demonstrate how this technique can incorporate the discretization approach and feature selection technique to improve the classification performance. Thus, this study can be summarized in three process as follows:

Discretization: This is the initial process that transform original datasets into discrete datasets. Two discretization techniques are used; K-Means and BkMD. Each discretization technique is applied to each dataset. At the end of discretization process, two discretize datasets are created. Now, three group of dataset will be used in the next process.

a) Original dataset called as ODS.

b) Discretized dataset with K-Means as discretization technique called as kMDS.

c) Discretized dataset with BkMD as discretization technique called as BkMDS.

Feature selection: This process will select the optimum feature in each dataset. Two techniques are used which are Information Gain (IG) and BA. 
Classification: In this paper, accuracy and sensitivity performance of classifiers over selected features has been made to compare the discretization and feature selection techniques by comparing their performances on original and discrete datasets. Two classifiers are used which are Naïve Bayes (NB) and k-Nearest Neighbor $(\mathrm{kNN})$. Each classifier is applied to each dataset.

Table 1. Dataset Information's

\begin{tabular}{cccc}
\hline$\#$ & Datasets & Number Of Instances & Number Of Attributes \\
\hline 1. & Credit Approval & 690 & 15 \\
2. & Ecoli & 336 & 8 \\
3. & Hill_Valley & 606 & 100 \\
4. & Image Segmentation & 210 & 19 \\
5. & Libras Movement & 360 & 90 \\
6. & Plant Species & 1600 & 64 \\
7. & Steel Plates Faults & 1941 & 27 \\
8. & Urban Land & 507 & 147 \\
9. & Automobile & 159 & 25 \\
10. & Abalone & 4177 & 8 \\
11. & Yeast & 1484 & 8 \\
12. & Waveform & 5000 & 21 \\
13. & Ionosphere & 351 & 34 \\
14. & Water Treatment & 523 & 38 \\
\hline
\end{tabular}

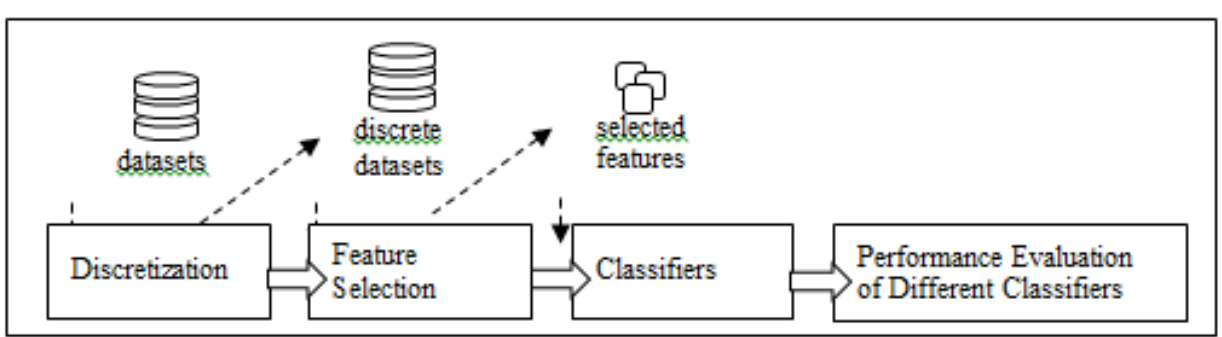

Figure 1. Framework of BkMDFS

\subsection{Bat-K-Means Discretization Technique, BkMD}

In BkMD, each of bat flies randomly with a velocity $v_{i}$, at position also known as solution $x_{i}$ with varying frequency or wavelength and loudness $A_{i}$. Generally, the frequency can be described as (1).।

$$
f=f_{\min }+\left(f_{\max }-f_{\min }\right) \beta
$$

where $\beta \in[0,1]$ is a random number drawn from a uniform distribution and $f \in[0,2]$ is a minimum and maximum value of frequency.

Therefore, the velocities $v_{i}^{t}$ and location $x_{i}^{t}$ can be updated according to the following (2) and (3), respectively.

$$
\begin{aligned}
& v_{i}^{t}=v_{i}^{t-1}+\left(v_{i}^{t-1}-v_{*}\right) f_{i}, \\
& x_{i}^{t}=x_{i}^{t-1}+v_{i}^{t},
\end{aligned}
$$

In this research, the initial value for $A$, is 0.5 . The aim of this technique is to searched and find its pray denote as feature that contribute to higher classification performance by changing the frequency, loudness and pulse emission rate $r$ by assumption the best solution is the bat can find the best feature in order to select the optimal feature from dataset. BA is used to generate a set of centroid, $C_{i}=\left(c_{1}, c_{2}, \ldots, c_{a}\right)$ for the each dataset that denote by (4). 


$$
\text { Fitness }=\sum_{1}^{N} u^{\wedge} 2
$$

where $u$ denote the data in dataset and $N$ attribute called as feature.

BA only handle in finding the optimize features. But, in discretization the essential thing is revolving the continuous domain into corresponding integer domain. Discretization is to set up the discrete range interval called as breakpoints for continuous attributes values in dataset. According to these breakpoints, the continuous attributes values are assigned into integer value such as 0,1,2 and 3 .

K-Means was described by J. MacQueen in 1967 [26]. K-Means is an iterative algorithms that begin with a set of $k$ reference point called as centroid [13]. First, data are partitioned into $k$ clusters. A data point $x$ become a member of cluster $k$-th if $k c$ is a centroid reference closet to $x$. The positions of centroid reference and the assignment of the data points to cluster is iterative computation and will be repeated until the optimal solution is obtained. K-Means can be define as (5).

$$
\sum_{k=1}^{K} \sum_{i \in S_{k}}\left\|x_{j}-k_{k} c\right\|^{2}
$$

where $K$ is the number of class $k$, which $G_{k}$ is a set of group, $G_{k}=\left(k_{1}, k_{2}, \ldots, k_{K}\right) . k_{k} c$ is the centroid for corresponding class, $k$-th. $x_{j}$ is $j$-th data point.

The strength of K-Means is able to transform from continuous to discrete value. However, K-Means not able to find the optimize features in the respective dataset. So, BA is used in order to cater this problem. In other word, K-Means and BA are compliment to each other's.

\subsection{BA Feature Selection Technique}

Each attribute in dataset correspond to feature in BA and each instance in dataset correspond to number of Bat in BA. Each instance or Bat have a set of attributes or features. Each feature has their own position that represented in binary bit strings with $N$-length, where $N$ is the total number of features. Each bits denotes as the feature which is ' 1 ' represent the selected feature and ' 0 ' represent unselected feature. The objective of BA is to find the Bat with minimum global best. Each bat will have their own local best and a set of binary string. Thus, a set of binary string with minimum local best be winner. This winner is called as global best. It means, the selected feature is corresponding to global best. For instance, from Table 2, there 4 bats in BA correspond to 4 instances in dataset and 5 features in BA correspond to 5 attributes in dataset. Each bat will have their own local best. Then, among the 4 local best, the local best = 0.111 is the minimum local best from bat number 2 . So, the winner bat is bat number 2 with global best, $g b=0.111$ and the selected feature, $f s=\{f 2, f 4, f 5\}$.

Table 2. Selected Features

\begin{tabular}{ccccccc}
\hline $\begin{array}{c}\text { \# of features, } f \\
\text { \# of bat }\end{array}$ & 1 & 2 & 3 & 4 & 5 & Local best \\
\hline 1 & 1 & 0 & 1 & 0 & 0 & 0.134 \\
2 & 0 & 1 & 0 & 1 & 1 & 0.111 \\
3 & 1 & 1 & 1 & 1 & 1 & 0.345 \\
4 & 1 & 1 & 0 & 0 & 0 & 0.543 \\
\hline
\end{tabular}

\subsection{Classification}

Classification process are used to measure the quality of selected feature from original dataset and discretize dataset. There are two supervised classifiers used: Naïve Bayes (NB) and k-Nearest Neighbor $(\mathrm{kNN})$ with two performance measures accuracy and sensitivity. Each classifiers is applied to each dataset.

\subsection{Performance Evaluation}

In this study, we propose BkMDFS that comprises discretization technique (BkMD) and feature selection technique (BA). To evaluate BkMDFS, we used two classifiers: Naïve Bayes (NB) and k-Nearest Neighbor (kNN) with two performance measures accuracy and sensitivity. Furthermore, as evident the capability of BA as discretization and feature selection techniques, there 6 other techniques as shown in 
Table 3 are employed. DPSO-RS was proposed by Noorhaniza in 2010 [25]. DPSO-RS was developed in order to improve PSO to cater discrete data DPSO-RS is hybrid with Rough Set to find the optimal feature selection problem. Each dataset is applied to each technique in Table 3. We have seven set of selected feature for each dataset, totally 98 set selected features. Then, classification process are used to measure the quality of selected features.

Table 3. Comparison Techniques

\begin{tabular}{cl}
\hline Technique & \multicolumn{1}{c}{ Description } \\
\hline BAO & Bat algorithm as a feature selection method and using ODS datasets. \\
IGO & Information Gain as a feature selection method and using ODS datasets. \\
kMB & Bat algorithm as a feature selection method using kMDS datasets. \\
kMIG & Information Gain as a feature selection method using kMDS datasets. \\
BkMDFS & Bat algorithm as a feature selection method using BkMDS datasets. \\
BkMDIG & Information Gain as a feature selection method using BkMDS datasets. \\
DPSO-RS & Discrete Particle Swarm Optimization - Rough Set that using ODS datasets. \\
\hline
\end{tabular}

\section{RESULTS AND ANALYSIS}

The experiments conducted to assess the robustness of proposed technique BkMDFS that comprised the discrete technique and feature selection technique together. 14 datasets from various domains have been used for experimental evaluation as shown in Table 1 .

Table 4 shows the accuracy for Naïve Bayes and k-Nearest Neighbor classifiers. BkMDFS techniques outperform 5 out of 14 datasets which are Ds1, Ds3, Ds5, Ds10 and Ds12 of the Naïve Bayes classifier. Meanwhile, BkMDFS technique only outperform in 4 datasets compare to IGO 7 datasets of the kNearest Neighbor classifier.

Table 5 shows the sensitivity for Naïve Bayes and k-Nearest Neighbor classifiers. BkMDFS techniques outperform 4 out of 14 datasets which are Ds1, Ds3, Ds5 and Ds12 of the Naïve Bayes classifiers. BkMDFS technique only outperform in 4 datasets compare to IGO 5 datasets for the k-Nearest Neighbor classifier.

From Figure 2, we focus results on the best discretization technique for each dataset, best classifier for each dataset and best combination of both for each type of dataset. As evident shows in Figure 2, BkMDFS outperform in all performance measure for Naïve Bayes classifier in discretize dataset. But, in sensitivity, BkMDFS also outperform in k-nearest neighbor classifier.

Table 4. The Performance of Accuracy

\begin{tabular}{|c|c|c|c|c|c|c|c|c|c|c|c|c|c|c|}
\hline \multirow[b]{2}{*}{ Dataset } & \multicolumn{7}{|c|}{ Naïve Bayes } & \multicolumn{7}{|c|}{ k-Nearest Neighbor } \\
\hline & BAO & IGO & kMB & kMIG & BkMDFS & BkMDIG & DPSO-RS & $\mathrm{BAO}$ & IGO & kMB & kMIG & BkMDFS & BkMDIG & DPSO-RS \\
\hline Ds1 & 0.628 & 0.783 & 0.733 & 0.752 & 0.852 & 0.842 & 0.808 & 0.609 & 0.827 & 0.733 & 0.756 & 0.809 & 0.849 & 0.804 \\
\hline Ds2 & 0.694 & 0.962 & 0.494 & 0.656 & 0.776 & 0.656 & 0.962 & 0.704 & 0.932 & 0.597 & 0.689 & 0.721 & 0.689 & 0.928 \\
\hline Ds3 & 0.515 & 0.519 & 0.253 & 0.253 & 0.522 & 0.253 & 0.519 & 0.589 & 0.584 & 0.496 & 0.496 & 0.59 & 0.496 & 0.586 \\
\hline Ds4 & 0.619 & 0.781 & 0.651 & 0.599 & 0.732 & 0.603 & 0.77 & 0.771 & 0.894 & 0.629 & 0.651 & 0.847 & 0.651 & 0.886 \\
\hline Ds5 & 0.626 & 0.647 & 0.479 & 0.371 & 0.661 & 0.345 & 0.65 & 0.816 & 0.865 & 0.638 & 0.591 & 0.871 & 0.412 & 0.867 \\
\hline Ds6 & 0.785 & 0.849 & 0.086 & 0.079 & 0.788 & 0.372 & 0.861 & 0.644 & 0.753 & 0.09 & 0.083 & 0.666 & 0.242 & 0.747 \\
\hline Ds7 & 0.616 & 0.667 & 0.505 & 0.54 & 0.64 & 0.623 & 0.616 & 0.642 & 0.713 & 0.55 & 0.566 & 0.724 & 0.575 & 0.679 \\
\hline Ds8 & 0.761 & 0.83 & 0.332 & 0.169 & 0.824 & 0.56 & 0.613 & 0.751 & 0.816 & 0.251 & 0.127 & 0.759 & 0.507 & 0.673 \\
\hline Ds9 & 0.486 & 0.562 & 0.091 & 1 & 0.494 & 0.507 & 0.569 & 0.717 & 0.855 & 0.091 & 1 & 0.786 & 0.557 & 0.799 \\
\hline Ds10 & 0.182 & 0.197 & 0.076 & 0.114 & 0.198 & 0.181 & 0.192 & 0.19 & 0.199 & 0.062 & 0.138 & 0.205 & 0.224 & 0.197 \\
\hline Ds 11 & 0.517 & 0.591 & 0.239 & 0.233 & 0.544 & 0.421 & 0.514 & 0.487 & 0.524 & 0.33 & 0.195 & 0.497 & 0.532 & 0.504 \\
\hline Ds 12 & 0.831 & 0.841 & 0.653 & 0.711 & 0.85 & 0.477 & 0.832 & 0.745 & 0.785 & 0.58 & 0.684 & 0.896 & 0.69 & 0.764 \\
\hline Ds 12 & 0.788 & 0.842 & 0.784 & 0.813 & 0.855 & 0.862 & 0.845 & 0.896 & 0.871 & 0.829 & 0.809 & 0.867 & 0.863 & 0.866 \\
\hline Ds14 & 0.613 & 0.739 & 0.351 & 0.56 & 0.725 & 0.56 & 0.723 & 0.563 & 0.699 & 0.326 & 0.522 & 0.633 & 0.522 & 0.679 \\
\hline
\end{tabular}

Table 4. The Performance of Sensitivity

\begin{tabular}{|c|c|c|c|c|c|c|c|c|c|c|c|c|c|c|}
\hline \multirow[b]{2}{*}{ Dataset } & \multicolumn{7}{|c|}{ Naïve Bayes } & \multicolumn{7}{|c|}{ k-Nearest Neighbor } \\
\hline & $\mathrm{BAO}$ & IGO & kMB & kMIG & BkMDFS & BkMDIG & DPSO-RS & $\mathrm{BAO}$ & IGO & kMB & kMIG & BkMDFS & BkMDIG & DPSO-RS \\
\hline Ds1 & 0.596 & 0.765 & 0.733 & 0.752 & 0.852 & 0.842 & 0.803 & 0.61 & 0.828 & 0.733 & 0.757 & 0.809 & 0.846 & 0.803 \\
\hline Ds2 & 0.735 & 0.973 & 0.565 & 0.67 & 0.786 & 0.67 & 0.973 & 0.711 & 0.932 & 0.64 & 0.696 & 0.735 & 0.696 & 0.935 \\
\hline Ds3 & 0.505 & 0.507 & 0.503 & 0.503 & 0.508 & 0.503 & 0.507 & 0.589 & 0.584 & 0.497 & 0.495 & 0.591 & 0.495 & 0.586 \\
\hline Ds4 & 0.619 & 0.786 & 0.6 & 0.571 & 0.729 & 0.605 & 0.776 & 0.771 & 0.895 & 0.586 & 0.638 & 0.848 & 0.648 & 0.886 \\
\hline Ds5 & 0.606 & 0.631 & 0.464 & 0.367 & 0.647 & 0.317 & 0.636 & 0.811 & 0.861 & 0.622 & 0.556 & 0.864 & 0.381 & 0.861 \\
\hline Ds6 & 0.774 & 0.842 & 0.084 & 0.094 & 0.781 & 0.357 & 0.851 & 0.639 & 0.739 & 0.083 & 0.112 & 0.658 & 0.228 & 0.737 \\
\hline Ds7 & 0.536 & 0.604 & 0.457 & 0.511 & 0.58 & 0.509 & 0.548 & 0.641 & 0.714 & 0.552 & 0.558 & 0.726 & 0.574 & 0.679 \\
\hline Ds8 & 0.75 & 0.821 & 0.369 & 0.232 & 0.815 & 0.589 & 0.548 & 0.738 & 0.804 & 0.25 & 0.232 & 0.738 & 0.476 & 0.672 \\
\hline Ds9 & 0.453 & 0.566 & 0.302 & 1 & 0.509 & 0.409 & 0.572 & 0.698 & 0.836 & 0.302 & 1 & 0.774 & 0.56 & 0.774 \\
\hline Ds10 & 0.233 & 0.241 & 0.156 & 0.155 & 0.236 & 0.204 & 0.232 & 0.192 & 0.201 & 0.053 & 0.155 & 0.207 & 0.258 & 0.198 \\
\hline Ds11 & 0.53 & 0.579 & 0.337 & 0.336 & 0.551 & 0.371 & 0.531 & 0.486 & 0.522 & 0.342 & 0.337 & 0.499 & 0.524 & 0.504 \\
\hline Ds12 & 0.814 & 0.81 & 0.551 & 0.649 & 0.84 & 0.368 & 0.806 & 0.746 & 0.785 & 0.584 & 0.685 & 0.889 & 0.691 & 0.764 \\
\hline Ds 12 & 0.798 & 0.826 & 0.775 & 0.803 & 0.843 & 0.863 & 0.829 & 0.895 & 0.863 & 0.821 & 0.798 & 0.86 & 0.863 & 0.858 \\
\hline Ds14 & 0.62 & 0.734 & 0.304 & 0.52 & 0.717 & 0.505 & 0.721 & 0.585 & 0.713 & 0.509 & 0.514 & 0.652 & 0.528 & 0.694 \\
\hline
\end{tabular}




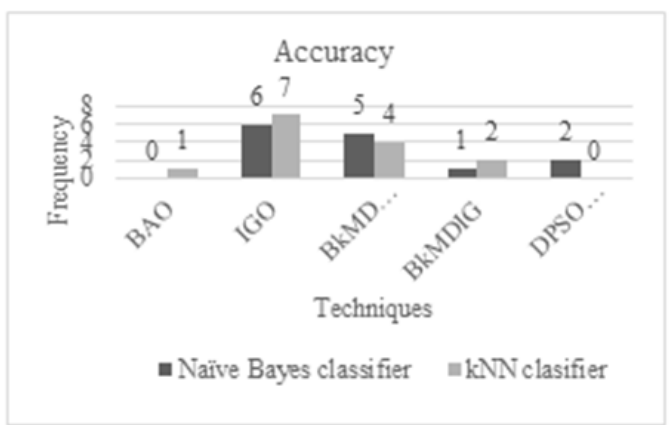

(a)

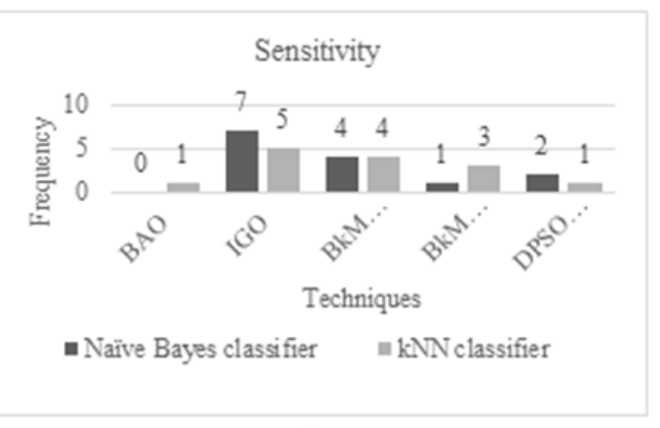

(b)

Figure 2. Number of dataset where each discretization technique yield the best

\section{CONCLUSION}

The objectives in this study is to investigate efficiency of the BA as a discretization and feature selection techniques. However, the drawback of BA is not able to transform the continuous into discrete value. So, we employ K-Means to solve this problem and proposed the discretization technique namely, BkMD. Then, to prove the capability BA as feature selection, we integrate BA with BkMD and known as, BkMDFS. As evident show the effectiveness of our proposed techniques, we run the experiment in 14 datasets from various applications. Two classifier are used to measure the quality of selected feature with BkMDFS which are Naïve Bayes and k-Nearest Neighbor.

In this study, the experiment has demonstrated that the discretization and Bat algorithm capable to correctly predict a class membership. BkMDFS able to improve classification performance (accuracy and sensitivity) in discretize dataset. Even though BkMDFS outperform in most of the comparison technique, but this technique still cannot compete with benchmark technique, information gain (IG). Thus, BkMDFS have a room for improvement in future research.

\section{ACKNOWLEDGEMENTS}

This paper has been supported by, Fundamental Research Grant Scheme (FRGS), Universiti Tun Hussein Onn Malaysia (UTHM) (vot 1612) for the financial support.

\section{REFERENCES}

[1] S. C. Tan, "Improving Association Rule Mining using clustering-based discretization of numerical data," in IEEE Press, Proc. 2018 International Conference on Intelligent and Innovative Computing Applications(ICONIC), 2018, pp. 1-5.

[2] K. Lavangnananda, S. Chattanachot, "Study of Discretization Methods in Classification," in IEEE Press, Proc. International Conference on Knowledge and Smart Technology, 2017, pp.50-55.

[3] K. Lavangnananda, and S. Waiwing, "Effectiveness of different pre-processing techniques on classification of various lengths of control charts patterns," Procedia Computer Science, vol. 69, pp. 44-54, 2015.

[4] D. García-Gil, et al., "Principal components analysis random discretization ensemble for big data," KnowledgeBased Systems, vol. 150, pp. 166-174, 2018.

[5] M.G. Rahman, and M. Z. Islam, "FIMUS: A framework for imputing missing values using co-appearance, correlation and similarity analysis," Knowledge-Based Systems, vol. 56, pp. 311-327, 2014.

[6] M. G. Rahman, et al., "Cairad: A co-appearance based analysis for incorrect records and attribute-values detection," in Neural Networks (IJCNN), The 2012 International Joint Conference on. Brisbane, Australia: IEEE, 2012, pp. 1-10.

[7] M. Sahu, et al., "Impact of discretization on classification of data using divide and conquer paradigm," in IEEE Press, Proc. International Conference on Electrical, Electronics, and Optimization Techniques, 2016, pp. 1596-1602.

[8] I. S. Thaseen and C. A, "Intrusion detection model using fusion of Chi square feature selection and multi class SVM," Journal King Saud University - Computer and Information Science, vol 29(4), pp. 462-472, 2017.

[9] J.Tang, et al., "Maximizing the spread of influence via the collective intelligence of discrete bat algorithm," Knowledge Based Syst, vol. 160, pp. 88-103, 2018.

[10] J. Lu, et al., "The continuous-discrete PSO algorithm for shape formation problem of multiple agents in two and three dimensional space," Applied Soft Computing, vol. 67, pp. 409-433, 2018. 
[11] D. Maryono, et al., "Implementation of Numerical Attribute Discretization for Outlier Detection on Mixed Attribute Dataset," in 2018 International Conference on Information and Communications Technology (ICOIACT), 2018, pp. 715-718.

[12] X. Xing, et al., "Continuous Attribute Discretization Algorithm of Rough Set based on k-means," in IEEE Press, Proc. 2014 IEEE Workshop on Advanced Research and Technology in Industry Applications (WARTIA), 2014, pp. 1384-1387.

[13] H. M. Tahir, et al., "Oving K-Means Clustering using discretization technique in Network Intrusion Detection System," in Computer and Information Sciences (ICCOINS), 2016 3rd International Conference, 2016, pp. 248-252.

[14] P. Shrivastava, et al., "A survey of nature imspired algorithms for feature selection to identify Parkinson's disease," Computer Methods and Programs in Biomedicine, vol. 139, pp. 171-179, 2017.

[15] B. Yang, et al., "Feature Selection Based on Modified Bat Algorithm," in IEICE Transactions on Information and Systems. E100.D. 2017, pp. 1860-1869.

[16] R. Cheruku, et al., "RST-BatMiner: A Fuzzy Rule Miner Integrating Rough Set Feature Selection and Bat Optimization for Detection of Diabetes Disease," International Journal of Engineering \& Technology Applied Soft Computing, 2017.

[17] H. Patel and M. Shah, "Rough set feature selection using Bat Algorithm", International Journal of Computer Science and Mobile Computing," vol 5(5), pp. 312-321, 2016.

[18] X.-S. Yang, "A New Metaheuristic Bat-Inspired Algorithm," Nature Inspired Cooperative Strategies for Optimization (NICSO 2010), vol. 284, pp. 65-74, 2010.

[19] E. Osaba, et al., "A discrete and improved bat algorithm for solving a medical goods distribution problem with pharmacological waste collection," Swarm and Evolutionary Computation, vol. 44, pp. 273-286, 2019.

[20] H. Li and S. Yin, "An improved discrete bat algorithm used for system fault diagnosis," Journal of Software Engineering, vol. 11, pp. 230-235, 2017.

[21] Y. Saji and E. Riffi, " A novel discrete bat algorithm for solving the travelling salesman problem," Neural Computing and Applications, vol. 27, pp. 1853-1866, 2015.

[22] E. Osaba, et al., "An improved discrete bat algorithm for symmetric and asymmetric traveling salesman problems," Engineering Applications of Artificial Intelligence, vol. 48, pp. 59-71, 2016.

[23] D. Rodrigues, et al., "A wrapper approach for feature selection based on bat algorithm and Optimum Path Forest," Expert Systems with Applications, vol 41 (5), pp. 2250-2258, 2014.

[24] A. C. Enache, et al., "Intelligent feature selection method rooted in Binary Bat Algorithm for intrusion detection," in IEEE 10th Junilee International Symposium on Applied Computational Intelligence and Informatics, 2015, pp. 517-521

[25] W. Noorhaniza et al., "Feature Selection Using a Novel Swarm Intelligence Algorithm with Rough Sets." in Proceedings of the 2010 International Conference on Data Mining (DMIN'10) Las Vegas, Nevada, USA (2010), 2010, pp. 294-300.

\section{BIOGRAPHIES OF AUTHORS}

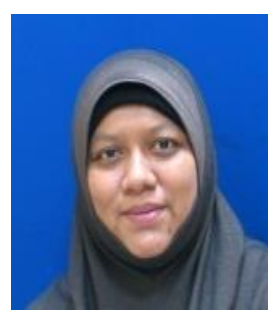

Rozlini Mohamed is a lecturer on information technology in Software Engineering department at the Universiti Tun Hussein Onn Malaysia. She has published a number of articles and proceedings in data mining. Her research interests include classification, heuristic algorithm, discretization and feature selection.

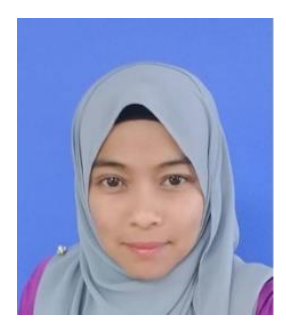

Munirah Mohd Yusof holds a Master from Universiti Utara Malaysia and her research interests are in knowledge based system, expert system, decision support system and e-learning. She is a lecturer of the Software Engineering Department in Universiti Tun Hussein Onn Malaysia. She has taught a number or courses in the field of Computer Science and has written and published several research papers in her area 

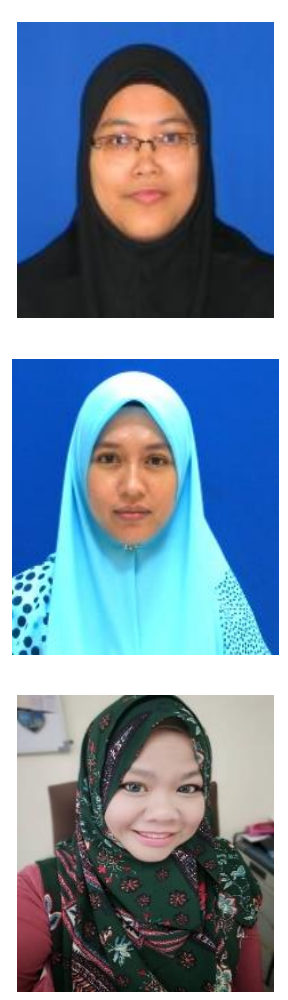

Noorhaniza Wahid is an Associate Professor in the Faculty of Computer Science and Information Technology, Universiti Tun Hussein Onn Malaysia. She received her $\mathrm{PhD}$ from the University of Sydney in 2011. Her research interests are in data mining and soft computing specifically in swarm intelligence algorithms. She also interested in multimedia application development in the area of virtual reality and augmented reality. She involved in teaching Computer Science and Multimedia courses for more than 20 years. She has written and published research papers in the area of data mining, soft computing, and multimedia. She also has been appointed as reviewers in many conferences and journals.

Norhanifah Murli holds a PhD from Auckland University of Technology and her research interests are in multimedia application development, data mining, spiking neural networks and fMRI data understanding and processing. She is a senior lecturer of the Multimedia Department in Universiti Tun Hussein Onn Malaysia. She has taught a number or courses in the field of Computer Science and has written and published several research papers in the area of data mining and multimedia.

Dr Muhaini Othman received her Bachelor in Information Technology from Northern University of Malaysia, Master in Computer Science from University of Malaya in 1999 and 2006 respectively and $\mathrm{PhD}$ in Computer and Mathematical Science from Auckland University of Technology in 2015. She is a Senior Lecturer at Faculty of Computer Science and Information Technology, Universiti Tun Hussein Onn Malaysia (UTHM). Her research interests include Personalised Predictive Modelling, Spiking Neural Networks and the representation of spatial and temporal data in ontology-based systems for personalised decision support. More information on Dr Muhaini can be found on FCSIT web site: http://fsktm.uthm.edu.my/v6/index.php/en/ 